\title{
A ROBOTIC STEPPER FOR RETRAINING LOCOMOTION IN SPINAL-INJURED RODENTS
}

\author{
D.J. Reinkensmeyer', W.K. Timoszyk ${ }^{1}$, R.D. de Leon ${ }^{2}$, R. Joynes ${ }^{2}$, E. Kwak', K. Minakata', V.R. Edgerton ${ }^{2}$ \\ 1: Department of Mechanical and Aerospace Engineering and Center for Biomedical Engineering \\ University of California, Irvine, 92697-3975
}
2: Department of Physiological Sciences and Brain Research Institute
University of California, Los Angeles, 90095-1760

\begin{abstract}
We describe the design and testing of a robotic system to assist locomotion training of spinal-injured rodents. The goal of the system is to control and quantify spatialtemporal patterns of movement and forces during the stance and swing phases of rat locomotion. This approach will allow us to provide varying levels of assistance to limb movement during stepping and to quantify the effects of assistance on step training. Our initial finding was that the rat spinal cord could perform stepping on a virtual treadmill generated by the robot system, with careful design of the virtual environment. Based on the conditions required for this "virtual stepping", we suggest several design principles for robot-assisted rehabilitative step trainers, including devices for humans.
\end{abstract}

\section{Introduction}

\subsection{Magnitude and Nature of Spinal Cord Injury and Locomotion Rehabilitation}

In the U.S. alone, over 10,000 people experience a traumatic spinal cord injury each year, and over 200,000 people with spinal cord injury are alive today [3]. Paralysis of the legs is common, resulting in loss of walking ability. Such paralysis is currently treated with intensive physical therapy, which traditionally includes strengthening of any residual voluntary control of muscles, maintenance of soft tissue compliance through stretching, and use of assistive technology such as wheelchairs to compensate for impairment.

Recently, an alternative approach to locomotion rehabilitation has shown promise [11]. The technique involves suspending a spinal cord injured subject in a harness above a treadmill and manually assisting the legs in moving in a walking pattern. The goal of this technique is to enhance residual locomotor control circuitry, thought to reside at least in part in the spinal cord. It is hypothesized that by providing appropriate sensory input (i.e. that associated with the remaining force, position, and touch sensors in the legs) during stepping in a repetitive manner that the spinal cord will learn to generate motor output appropriate for stepping.
This training approach is based on studies of spinaltransected animals that suggest that the spinal cord can learn a motor task without input from the brain. This research showed that spinal animals are capable of generating rhythmic locomotion activity while fully bearing their weight. Spinal cats that do not receive treadmill training generally recover only $25 \%$ of the stepping capability that trained cats acquire $[1,6]$. When training is not maintained for several weeks, stepping ability declines, further demonstrating the use dependent acquisition of stepping [2].

Clinical trials of treadmill training with humans are just beginning in the U.S., while research into the neurophysiological bases of treadmill training with animal models such as the spinal rat is accelerating. A current limitation in both the human and animal research, however, is that there is poor experimental control over step training, and quantification of the motor patterns is difficult and time consuming. For example, in treadmill training with humans, three therapists are often needed for each patient, one for each leg of the patient, and one to manipulate the patient's hips. The required patterns of forces applied by the therapists are undetermined. Manual assistance of the limbs of a rat during treadmill training is even more difficult to achieve and also poorly understood, in part because manipulating small limbs cannot be performed in a consistent manner.

\subsection{Potential Role of Robotics}

Recently there has been a surge of interest in bringing advances in robotic and mechatronic technology to bear on neurologic rehabilitation [9]. Initial research has focused primarily on devices for providing therapy to the arm after stroke [5,7-10], since stroke is the leading cause of major disability in the U.S., and arm impairment after stroke is common. However, treadmill training after spinal cord injury also provides an intriguing target for robotic technology. Specifically, robotic technology could improve experimental control over treadmill training, thus providing a means to better understand and optimize its effects. Robotic technology could also provide a means to quantify in real-time the kinematics and kinetics of stepping. Ultimately, robotics could also 
provide a means to both automate and monitor treadmill training in the clinic.

The work reported here is a first attempt to use robotics to enhance locomotor training in the spinal rat. Although our ultimate goal is to develop a device for step training of humans (cf. [4]), there are at least three compelling reasons to develop a rat robot also. First, as mentioned above, such a robot could provide improved experimental control and measurement for treadmill training in rats, enhancing basic research capability. Second, development of a rat stepper could provide useful data for development of a device for humans. In effect, a rat robot could serve as a small-scale, simplified test-bed for evaluating the physiological and engineering principles to be used in the human robotic stepper. Third, the benefits of robotic technology may also extend to other repair strategies such as regeneration and cell transplants. For example, it is possible that precisely controlled patterns of sensory input could provide "directional" guide for regenerating fibers so that the appropriate target neurons would be more likely not only to develop new connections, but also to make appropriately functional connections. Novel regeneration therapies intended for coupling with locomotor training tested in animal models such as the rat are likely to significantly facilitate the development of assistive devices for humans.

\section{Design Considerations}

\subsection{Design Objective and Questions}

Our overall design objective is to provide a means to control and quantify spatial-temporal patterns of movement and force during the stance and swing phases of rat locomotion, in order to optimize spinal recovery of stepping. However, at the onset of this project, several questions essential for meeting this goal were unknown, including:

What time-sequence of forces/movements from the hind limb does the spinal cord need to experience in order to best recover stepping ability? Our working perspective of the spinal cord is that it performs pattern recognition that facilitates the generation of stepping. Specifically, a distributed sensor array comprised of hundreds of biological touch, position, and force sensors reports to the spinal cord about the limbs' physical state and environmental interactions. If the sensor array reports a specific pattern of sensory input consistent with locomotion, the spinal cord recognizes this pattern, and generates motor output for stepping. Repeated application of this pattern is hypothesized to strengthen the associated motor output. Rational design of a robot step trainer thus depends on a clear understanding of the optimal sequence of sensory input to be provided to the spinal cord. This in turn, however, is a key unanswered question the stepper itself is intended to address.

A second, related question was:

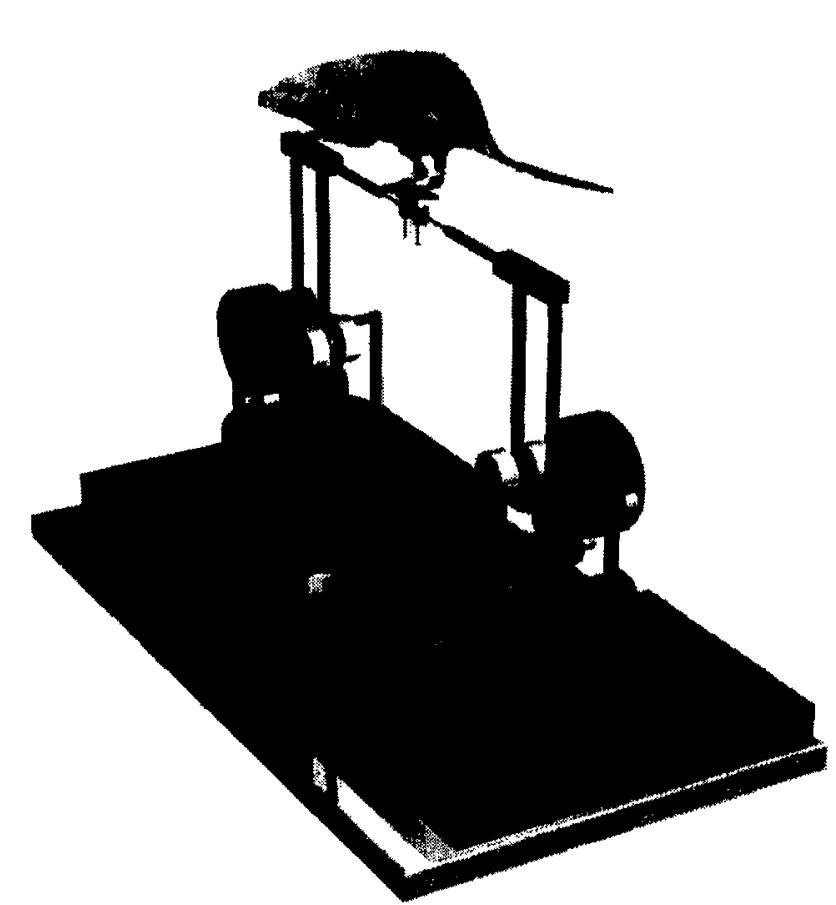

Figure 1: The Rodent Stepper. Two PHANToM 1.0 robots attach to the rat's paws. The robots simulate a virtual treadmill. The rat's body was manually held above the virtual treadmill during stepping.

What are valid robot/hind limb contacts for generating stepping? During normal stepping the rat walks on its toes. It thus makes physical contact with its environment only during stance through the bottom of its toes. Physical contact to the hind limb at other locations and times can elicit a variety of spinal-modulated reflexes. For example, if the top of the paw contacts an obstacle during swing, the limb may flex to clear the barrier. Thus, a key question was where to attach to the rat's hind limb during stance and swing. Would attaching a robot to the hind limb during swing inhibit swing?

\subsection{Design Approaches}

As a starting point, we determined the stepper should be able to at least emulate a treadmill, while also providing a means to apply force to the hind limbs during swing. We identified two potential configurations for the robot stepper:

a) Integrate a robot with a treadmill, and attach the robot to the rat's heel or ankle, such that the rat can place its toes on the treadmill while the robot can apply forces and measure the kinematics of the attachment point.

b) Replace the treadmill with a robot, attach the robot to the toes, and generate a virtual treadmill through haptic simulation (Fig. 1).

We chose to investigate the second approach first for several reasons. First, designing a virtual treadmill was simpler, requiring only development of robots instead of 
integration of robots with a treadmill. Second, by using the robot to provide support for the hind limb during stance, the robot itself can quantify the contact force against the foot, via knowledge of the robot actuator forces. Third, using a virtual treadmill would allow arbitrary variation of treadmill properties (eg. surface shape, velocity profile, friction, stiffness), and thus allow systematic examination of how these properties affect step generation. However, a disadvantage of the virtual treadmill approach is that the robot attachment point is identical during stance and swing, possibly interfering with swing. Integrating a robot with a physical treadmill would allow attachment at the heel or ankle during swing, while still achieving loading at the toes during stance.

\section{Design and Testing}

This section describes the iterative design and testing process for the robot-based virtual treadmill. Our efforts have initially focused on confirming that a spinal rat capable of stepping on a physical treadmill can also step on the virtual treadmill.

\subsection{Description of the Robots and Rodents}

Robots: Two commercially available robotic arms were chosen to implement the virtual treadmill (Fig. 1). The robotic arms are the PHANToM 1.0 haptic interfaces commercialized by SensAble Technologies, Inc. They are small, cable-driven mechanical linkages that provide high fidelity three degrees of freedom force control. They were primarily designed to provide a sense of touch in the manipulation and deformation of computer generated 3D objects with the hand and fingers. However, the high fidelity of their actuation and sensing, coupled with their low inertia and friction, made them attractive as off-theshelf manipulators for the rat hind limb. Additionally, the robots have a software development kit, the General Haptic Open Software Toolkit (GHOST SDK 2.1), which allows for the programming of a variety of virtual objects, as well as direct specification of motor forces.

Rats: Experiments were performed with four rats completely transected at the mid-thoracic level, paralyzing the hind limbs. Transections were performed five days after birth, as a more robust recovery of stepping occurs when transections are performed shortly after birth. At the time of the experiments described below, the rats were one to two months old, and were capable of stepping on a physical treadmill due to intrinsic recovery in the spinal cord, although this stepping was sporadic and impaired (e.g. the rats often failed to initiate swing). All experiments were performed under the guidelines of the Animal Use Committee at UCLA.

\subsection{Design Iteration 1}

Initial Design: Our approach in simulating a treadmill was to create a virtual block moving in the horizontal plane. Using the GHOST gstFreebody class, the block was programmed to move at a constant velocity, and its surface properties were set to emulate static and dynamic friction. The stiffness and damping of the block in the vertical plane was set to $1.0 \mathrm{~N} / \mathrm{mm}$ to $0.005 \mathrm{~N} / \mathrm{m} / \mathrm{s}$. The dynamic and static friction coefficients were set at their maximum allowable value of 1.0 .

The factory-shipped end-effector of the PHANToM robots consists of a gimbal that can be attached to a pen or thimble. In order to connect the robots to the rats, two rectangular platforms the size of the rats' toes (approximately $1 \mathrm{~cm}$ by $1 \mathrm{~cm}$ ) were fabricated from PC board, and attached to the gimbal.

Experimental Results: In the first experimental session, two spinal rats were tested on the virtual treadmill. As mentioned above, both rats were capable of stepping on a physical treadmill, albeit inconsistently, due to intrinsic recovery in the spinal cord that had occurred since transection. The rats' toes were attached to the platforms with $1.5 \mathrm{~cm}$ wide adhesive tape (Fig. 2). The rats' bodies were then manually held such that the feet contacted the virtual treadmill.

Our finding was that the hind limbs flexed as the body was lowered toward the treadmill, and the treadmill would not engage to drive the hind limbs backward. By placing weights on the platforms, it was determined that at least $17 \mathrm{gm}$ of downward force were required to engage the treadmill, owing to the friction in the robot resisting both downward and backward movement. When the rat's hind limb was manually extended to engage the treadmill, it

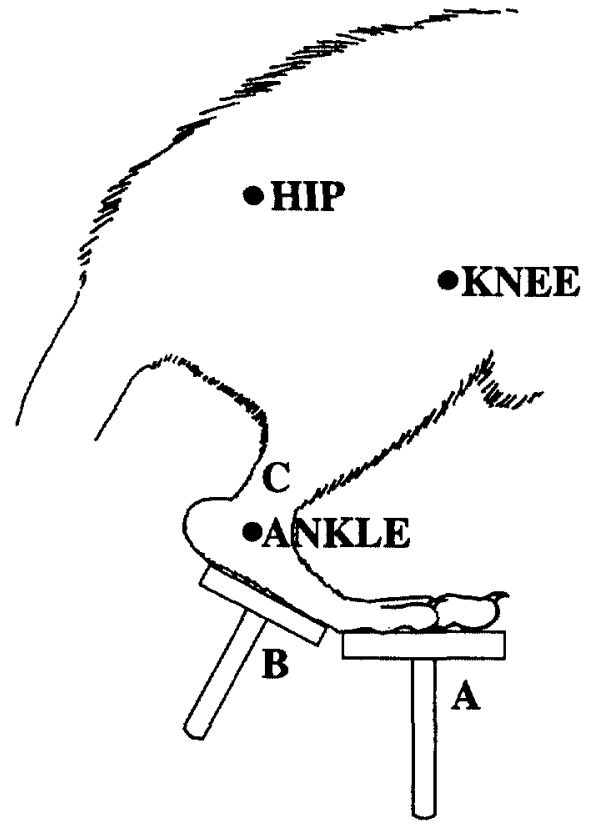

Figure 2: Robot/hind limb contact locations. For design iterations 1 and 2, the platform was taped to the toes (A). For design iteration 3, it was taped at the heel (B). For the tape experiment, small pieces of tape were applied at the toes (A), heel (B), and ankle (C). 
would sometimes generate a swing-like flexion movement.

Other problems with the initial design also became clear. The weight of the gimbal itself was enough to invert the rats' ankles, resulting in an abnormal walking position. The gimbal's degrees of freedom were thus removed by constraining them with adhesive tape. Also, when a swing was manually induced, the rats' hind limbs would sometimes move close to each other, and the robots would physically interfere with each other and disrupt cyclic movement.

\subsection{Design Iteration 2}

Design Modifications: Based on this experience, several changes were made in the virtual treadmill design. The need for increased friction was addressed by replacing the existing virtual treadmill controller with a positiondependent velocity controller. Now, when the hind limb extended at or below the plane of the virtual treadmill, the robot moved backwards under velocity control, regardless of the contact force exerted against the virtual treadmill surface. A virtual, vertical planar constraint was also installed for each hind limb so that the hind limbs were restricted to preset planes and could not interfere with each other. Additionally, the gimbal frame was counterbalanced, such that its weight would not apply moments to the rats' paws.

Experimental Results: The modified system was tested in a second experimental session with a single rat selected because it performed stepping on a physical treadmill most consistently of the four available rats. When the rat's toes were taped to the pads, the rat was able to engage the treadmill. However, the counterbalanced gimbals did not prevent the rat from inverting/everting or supinating/pronating its foot. The gimbal's degrees of freedom were thus removed again using tape.

With added foot stability, the rat was able to achieve rhythmic stepping (Fig. 3). However, this stepping was sporadic, as the rat would step for periods ranging from only one to two minutes, then fail to step consistently for longer periods of time. The planar constraints were useful in preventing collision of the hind limbs during stepping.

Figure 3 shows kinematic and kinetic data from a sixsecond window selected from a period of approximately two minutes during which the rat stepped consistently. The hind limbs moved in an alternating gait, as can be seen in the vertical contact force generated against the robot during stance. The hind limbs stepped with unequal stride length, with one hind limb consistently returning to a more flexed position (Fig. 3). Both hind limbs often slowed dramatically during swing, then dropped quickly to the treadmill (Fig. 4).

Although virtual stepping was achieved in this second experiment, the stepping was difficult to evoke compared to stepping by the same rat on a physical treadmill. In particular, the rat often failed to initiate swing. Two possible causes of this failure were: 1) the robot/hind limb contact produced inappropriate sensory input that inhibited swing; and 2) the inertia and friction properties of the robot retarded swing. These possibilities were evaluated in two further experiments.

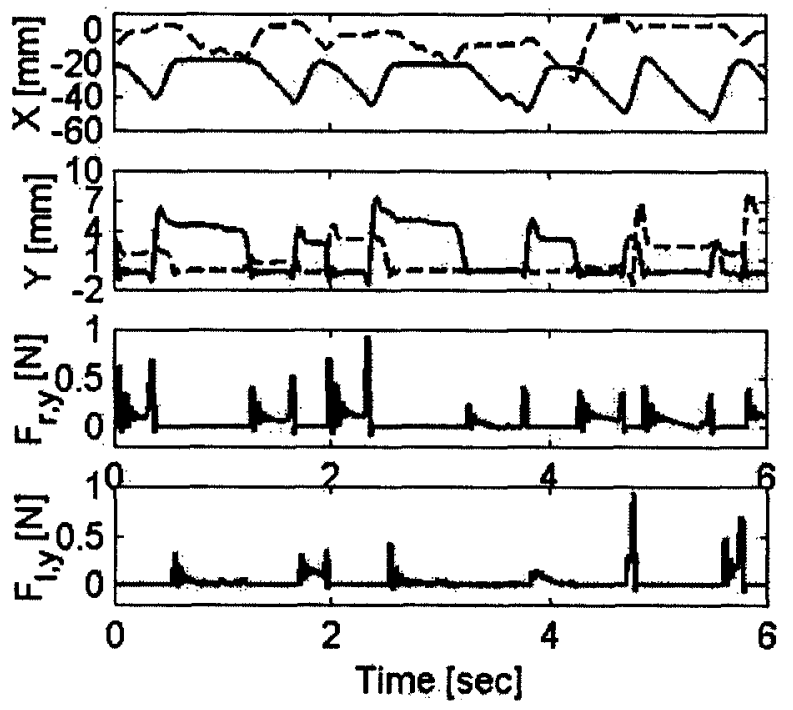

Figure 3: Paw movement and loading during virtual stepping for design iteration 2. X: horizontal movement. $Y$ : vertical movement. Dashed line: left hind limb. Solid line: right hind limb. $\mathrm{F}_{\mathrm{r}, \mathrm{y}} \mathrm{F}_{\mathrm{l}, \mathrm{y}}$ : upwards force applied by robot to right and left hind limbs, respectively. The treadmill moved in the $-X$ direction at $7 \mathrm{~cm} / \mathrm{sec}$. Note alternating stance in the force traces.

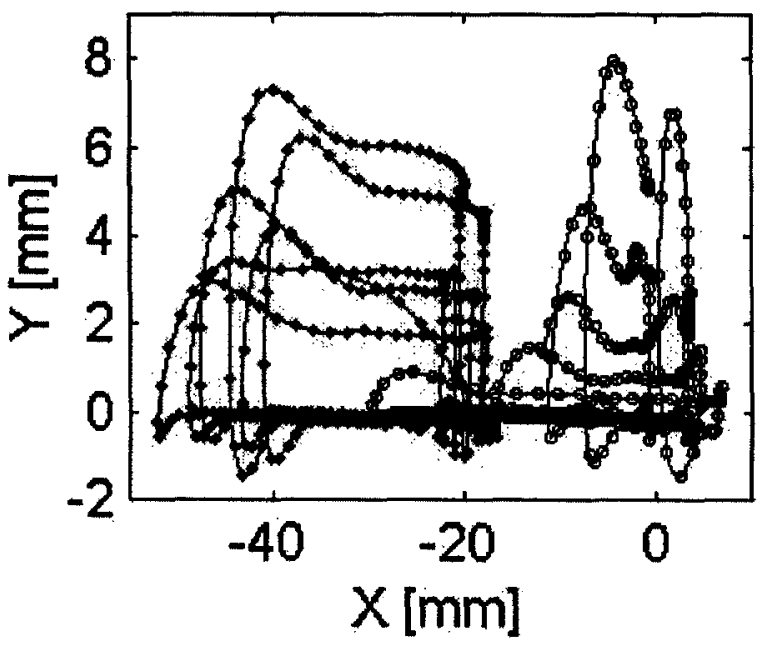

Figure 4: Paw movement in the saggital plane during virtual stepping for design iteration 2. The treadmill roved to the left ( $-X$ direction). Open circles: right hind 'mb; closed circles: left hind limb. 


\subsection{Evaluation of Robot/Hind Limb Contact Location}

The first experiment was designed to determine the effect of robothind limb contact location on stepping consistency. Adhesive tape $(1.5 \mathrm{~cm}$ wide, $2.0 \mathrm{~cm}$ long) was wrapped around the lower extremity of four spinal rats, at one of three locations: around the toes (as was done in attaching the rats to the virtual treadmill in the first two experiments), around the heel, and around the ankle (Fig. 2). The rats were then held manually on a physical treadmill, and their stepping ability was videotaped. For each of the four rats, the three tape locations, as well as the condition of no tape on the hind limb, was randomly varied. The number of successful steps completed in a thirty-second period was determined later off-line using video analysis for each rat. Without tape 34 steps \pm 11 (mean \pm standard deviation) were made; with tape on toes, $11 \pm 9$ steps; with tape on ankle, $8 \pm 9$ steps; and with tape on heel: $28 \pm 12$ steps. These results strongly suggested that contacting the rat at the toes (or ankle) decreased stepping consistency, potentially accounting for difficulties in evoking stepping on the virtual treadmill.

\subsection{Design Iteration 3}

Design Modifications: Based on these results, it was determined to compare virtual stepping with the robots attached at the toes with virtual stepping with the robots attached at the heel. Several changes were also made to the virtual treadmill. The gimbal was removed and replaced with a fixed joint. Additionally, control software was developed in order to reduce the inertia and friction of the robots during swing.

To compensate for inertia, the inertia of the robots was first estimated by assuming the robot acted as a simple mass in the workspace region in which stepping occurred. To identify this mass, the robot endpoint was moved in a sinusoidal trajectory in the horizontal and then vertical plane. The motor forces were recorded, and the acceleration of the endpoint was calculated by doubledifferencing the position trajectory. Acceleration was plotted against motor force, and the linearized inertia of the robot was estimated using the slope of this relationship. The estimated inertia was $42 \mathrm{gm}$ in the vertical plane, and $75 \mathrm{gm}$ in the horizontal plane.

To estimate static friction, the motors were programmed to apply a ramp force with the robot in the center of the stepping workspace, and the force at which the robot began moving was measured. The static friction in the horizontal and vertical planes was approximately $0.14 \mathrm{~N}$ and $0.05 \mathrm{~N}$ respectively.

Based on these estimates, the robots were programmed to apply assistive forces to compensate for inertia and friction. The inertia-compensating force was calculated by multiplying the estimated inertia by the robot endpoint acceleration, low pass filtered at $6 \mathrm{~Hz}$. With this simple approach, the robot remained stable with compensation of up to $60 \%$ of the estimated inertia. The frictioncompensating force was equal to the estimated static friction, applied in the direction of motion of the robot endpoint.

Experimental Results: Stepping ability was first tested with the robots attached to the rat's toes, using the friction and inertia compensation. As in the previous experiment, short sequences of steps could be elicited, but alternating gait was not sustained for more than several strides over an hour testing period. The robots were then reattached to the rats' heels, using the same small rectangular platforms and adhesive tape (Fig. 2). Longer sequences of consistent stepping were achieved. The rat stepped with smooth swing trajectories and near normal stride-lengths (Fig. 5 and 6) for up to twenty strides. However, even more consistent stepping sequences were achieved when the rat stepped on a physical treadmill without the robots attached.

\section{Summary and Conclusions}

These results demonstrate that the rat spinal cord can perform stepping in a virtual environment generated by robots, with careful consideration of the design of the virtual environment. Future work will investigate how this virtual environment can be used to facilitate step training. For example, control algorithms for adaptively assisting in swing of the hind limb may be useful in training stepping. The possibilities for applying controlled manipulations to the limbs are diverse. Coupled with the ability to quantify in real-time stepping kinematics and loading, this improved control ability will provide a powerful tool for advancing understanding of spinal control of locomotion and regeneration.

The results reported here also suggest several design principles that may be useful in rational design of other robot-assisted step trainers, including human devices. First, the spinal cord-injured hind limbs were weak, requiring careful design of the impedance of the virtual environment in which they stepped. For example, the friction properties of the virtual treadmill were increased to better engage the hind limb during stance. Also, the inertia and friction properties of the robot likely altered the swing trajectory (cf. Figs. 4 and 6). Future research will investigate more fully the effect of robot inertia and friction on swing kinematics.

A second finding was that body degrees of freedom that were poorly controlled by the spinal cord needed to be constrained. For example, the gimbal was removed in order to hold the foot in a configuration appropriate for stepping, and virtual vertical, planar constraints were used to prevent the hind limbs from physically interfering with each other during stepping. Distinguishing the body degrees of freedom that require constraint, from those that 
need freedom to generate consistent stepping, will likely also be a key problem in the design of human step trainers (consider for example the six degree-of-freedom movements of the torso during normal human walking).

Lastly, robot/ hind limb contact location mattered. Contacting at the heel rather than at the toes resulted in an improvement in stepping. We hypothesize that contacting at the toes during swing inhibits swing because the spinal cord associates sensory input from the toes with stance. Future research will investigate the alternate design
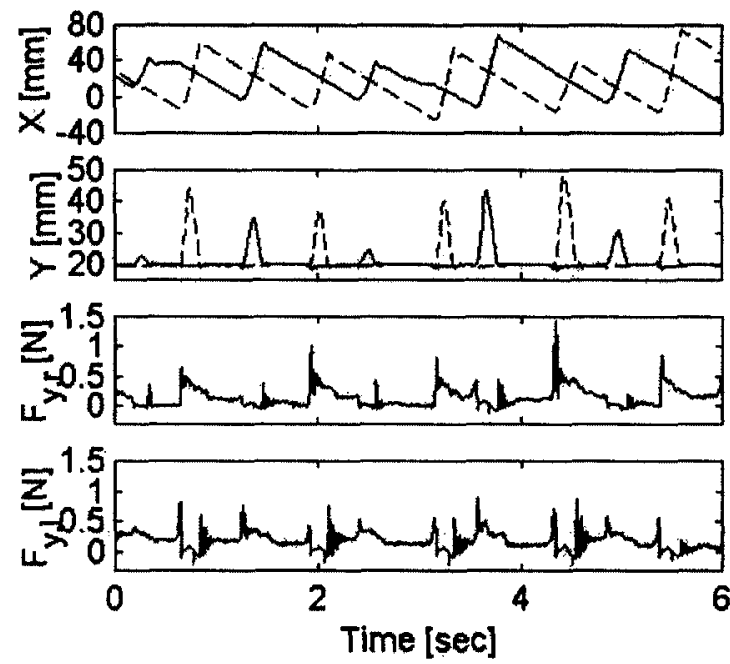

Figure 5: Paw movement and loading during virtual stepping for design iteration 3 (robots attached at the heels). X: horizontal movement. Y: vertical movement. Dashed line: left hind limb. Solid line: right hind limb. $F_{r, y}, F_{1, y}$ : upwards force applied by robot to right and left hind limbs, respectively. These forces were nonzero during swing because of the inertia/friction compensation. The treadmill moved in the $-\mathrm{X}$ direction at $7 \mathrm{~cm} / \mathrm{sec}$.

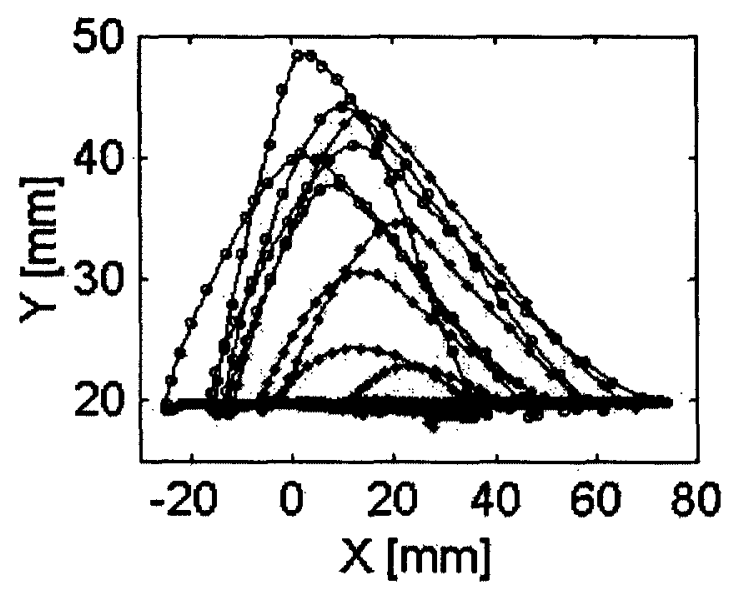

Figure 6: Paw movement in the sagittal plane during virtual stepping for design iteration 3 (robots attached at the heels). The treadmill moved to the left. Open circles: left hind limb, closed circles: right hind limb. approach of using a physical treadmill integrated with the robots (attached at the heel or ankle) as described in Section 2.2. This approach would allow sensory input to the toes during stance but not during swing, while maintaining the ability to measure and assist in hind limb movement.

As mentioned above, our working perspective of the spinal cord is that it performs sensory pattern recognition that in turn facilitates step generation. The results with the rodent stepper suggest that in doing pattern recognition, the spinal cord pays considerable attention to details. If those details are inconsistent, it will not facilitate stepping. Robot devices for step training will need to be well versed in the relevant details.

\section{References}

[1] R. D. de Leon, J. A. Hodgson, R. R. Roy, and V. R. Edgerton, "Locomotor capacity attributable to step training versus spontaneous recovery following spinalization in cats," J. Neurophysiology, vol. 79, pp. 1329-1340, 1998.

[2] R. D. de Leon, J. A. Hodgson, R. R. Roy, and V. R. Edgerton, "The retention of hindlimb stepping ability in adult spinal cats after the cessation of step training," $J$. Neurophysiol., vol. 81, pp. 85-94, 1999.

[3] B. H. Dobkin, Neurologic Rehabilitation.

Philadelphia: F.A. Davis Company, 1996.

[4] A. Bejczy, "Towards development of robotic aid for rehabilitation of locomotion-impaired subjects," Proc. $I^{\text {st }}$

Workshop Robot Motion and Control (RoMoCo'99)

Kiekrz, Poland, pp. 9-16, 1999.

[5] H. I. Krebs, N. Hogan, M. L. Aisen, and B. T. Volpe, "Robot-Aided Neurorehabilitation," IEEE Trans. Rehab. Eng., vol. 6, pp. 75-87, 1998.

[6] R. G. Lovely, R. J. Gregor, R. R. Roy, and V. R. Edgerton, "Effects of training on the recovery of full weight-bearing stepping in the adult spinal cat," Exp. Neurol., vol. 92, pp. 421-435, 1986.

[7] P. S. Lum, C. G. Burgar, D. Kenney, and H. F. M. Van der Loos, "Quantification of force abnormalities during passive and active-assisted upper-limb reaching movements in post-stroke hemiparesis," IEEE Trans Biomedical Eng, vol. 46, no. 6, pp. 652-662, 1999.

[8] P. S. Lum, H. F. M. Van der Loos, P. Shor, and C. G. Burgar, "A robotic system for upper-limb exercises to promote recovery of motor function following stroke," Proceedings Sixth Int. Conf. on Rehab. Robotics, pp. 235239, 1999.

[9] D. J. Reinkensmeyer, N. Hogan, H. I. Krebs, S. L. Lehman, and P. S. Lum, "Rehabilitators, robots, and guides: New tools for neurological rehabilitation," in Biomechanics and Neural Control of Movement, $\mathrm{J}$. Winters and P. Crago, Eds.: Springer-Verlag, 2000. [10] D. J. Reinkensmeyer, J. P. A. Dewald, and W. Z. Rymer, "Guidance based quantification of arm impairment following brain injury: A pilot study," IEEE Trans on Rehab Eng, vol. 7, pp. 1-11, 1999.

[11] I. Wickelgren, "Teaching the Spinal Cord to Walk," Science, vol. 279, pp. 319-321, 1998. 